\title{
OPEN Development, evaluation of the PNA RT-LAMP assay for rapid molecular detection of SARS-CoV-2
}

\author{
Chinbayar Bat-Ochir ${ }^{1,3 \bowtie}$, Yeon-Sook Kim ${ }^{2,3}$, Han Gyeul Kim ${ }^{1}$, Si Seok Lee ${ }^{1}$, Han Woo Lee ${ }^{1}$ \& \\ Hee Kyung Park ${ }^{1}$
}

Dual-labeled PNA probe used RT-LAMP molecular rapid assay targeting SARS-CoV-2 ORF1ab and $\mathrm{N}$ genes was developed, and the analytical, clinical performances for detection of SARS-CoV-2 RNA extracted from clinical nasopharyngeal swab specimens were evaluated in this study. Data showed that this assay is highly specific for SARS-CoV-2, and the absolute detection limit is 1 genomic copy per microliter of viral RNA which can be considered to be comparable to gold-standard molecular diagnostic method real-time reverse transcriptase PCR. Both clinical sensitivity and specificity against a commercial real-time RT-PCR assay were determined as identical. In conclusion, the PNA RT-LAMP assay showed high analytical and clinical accuracy which are identical to real-time RT-PCR which has been routinely used for the detection of SARS-CoV-2.

Severe Acute Respiratory Syndrome Coronavirus 2 (SARS-CoV-2) is a single-stranded RNA virus that causes Coronavirus Disease 2019 (COVID-19) which has been spreading globally at rapid speed and is more contagious than most of other human respiratory tract infectious microorganisms ${ }^{1,2}$. The high transmissibility demands rapid and accurate detection of SARS-CoV-2 at the early stages of the infection in a cost-effective manner ${ }^{3}$. Highly specific real-time reverse transcriptase PCR assays have been used for the identification of the SARS-CoV-2 RNA globally; however, it requires laboratory-based PCR instruments and needs about $2 \mathrm{~h}$ of run-time as well as additional incubation of 15 to 30 min for cDNA synthesis from template RNA ${ }^{4}$.

Loop Mediated Isothermal Amplification (LAMP) is a molecular technique capable of detecting nucleic acids with high sensitivity within a reduced time compared to classical real-time PCR and has been used widely for the detection of viral infections in a time-effective manner ${ }^{5}$. Since beginning of the COVID-19 outbreak, various molecular detection assays based on real-time PCR or LAMP aimed for detection of SARS-CoV-2 nucleic acid have been developed and received Emergency Use Authorizations (EUA) from the US FDA or the authorizations of the regulatory agencies in the country of production. However, quite a few LAMP tests have been commercialized successfully and multiple scientific reports have shown that the LAMP tests have high enough analytical and clinical performances ${ }^{6-8}$, numerous reports have been showing that the LAMP assays still have some challenges; for instance, poor specificity ${ }^{9,10}$, difficulties of establishing multiplexed testing ${ }^{11,12}$, and result interpretation through colorimetric/visual inspection which might be affected by technicians' subjectivity ${ }^{13}$. Notably, SARSCoV-2 LAMP tests have shown inadequate detection performances when testing low positive samples which exhibited Ct values over 30 on real-time PCR assays ${ }^{14-16}$; consequently, LAMP has been considered that having similar diagnostic accuracy as real-time PCR in detection of SARS-CoV-2 in the acute symptomatic phase of COVID-19 while the sample contains high viral load but not in early or late/clearance stages of the infection ${ }^{17,18}$.

To solve those challenges, we developed a Peptide Nucleic Acid based Real Time-LAMP (PNA RT-LAMP) assay "AQ-TOP COVID-19 rapid Detection Kit Plus" which used dual labeled PNA probe that has been reported having superior specificity ${ }^{19}$ and sensitivity ${ }^{20}$ comparing to the accumulative dye such as SYBR green or sequence specific DNA fluorescent probes which are routinely used in LAMP and other molecular assays.

PNA is an artificial analog of DNA which the negatively charged phosphodiester backbone is replaced by a neutrally charged N-(2-aminoehtyl)-glycine backbone. The neutrally charged backbone makes PNA probe to bind its complementary nucleic acid with much higher affinity than the DNA detection probes, consequently,

\footnotetext{
${ }^{1}$ Seasun Biomaterials, Daejeon, Korea. ${ }^{2}$ Division of Infectious Diseases, Department of Internal Medicine, Chungnam National University School of Medicine, Daejeon, Korea. ${ }^{3}$ These authors contributed equally: Chinbayar Bat-Ochir and Yeon-Sook Kim. ${ }^{\square}$ email: chinba@seasunbio.com
} 


\begin{tabular}{|l|l|l|l|}
\hline Target & Oligonucleotide & Query with mismatch against 56,303 SARS-CoV-2 gRNA & Predicted inclusivity \\
\hline \multirow{2}{*}{ ORF1ab } & Primers $(6$ each) & 0 & \multirow{2}{*}{$100 \%$} \\
\cline { 2 - 5 } & Probe $(1$ each $)$ & 0 & \multirow{2}{*}{$100 \%$} \\
\hline \multirow{2}{*}{ N gene } & Primers $(6$ each) & 0 & \\
\cline { 2 - 4 } & Probe $(1$ each $)$ & 0 & \\
\hline
\end{tabular}

Table 1. Summary of oligonucleotide sequence analysis. Sequences of all primer and probes showed no mismatch against the target queries (SARS-CoV-2 whole genomic sequences).

the sensitivity of PNA probe against the target nucleic acid is reported to be higher than the DNA probe ${ }^{21,22}$. Furthermore, the specificity of the PNA is enhanced due to absence of the inter strand repulsion between PNA and its target nucleic acid $^{23}$. Those particular biochemical properties make PNA suitable for use in many biological applications, especially as detection probes for PCR, FISH, multipurpose microarrays, and biosensors ${ }^{24-27}$.

In this study, the analytical and clinical performances of the PNA RT-LAMP assay against commercially available real-time PCR and colorimetric LAMP assays targeting SARS-CoV-2 were evaluated on both benchtop and portable real-time molecular amplifiers.

\section{Results}

Establishment of the PNA RT-LAMP assay. Two (2) sets of primer and PNA probes targeting two (2) specific regions in ORFlab and N gene of SARS-CoV-2 were designed for amplification and detection of SARS-CoV-2. The sequences of the oligonucleotides were aligned against publicly available 56,303 SARS-CoV-2 sequences downloaded from NCBI database by February 2021 which contain full genomic information on CLC Main Workbench. All alignments showed $100 \%$ of identity against the queries showing that in silico analysis predicted that the assay can amplify and detect all SARS-CoV-2 isolates analyzed in this study. Results are summarized in Table 1, primer and probe sequences are shown in Supplementary Table S1.

The assay targets one (1) specific region in each ORF1ab and N gene of the SARS-CoV-2 in two separate tubes with FAM fluorescence channel. Each tube contains primer and probe targeting human RNase $\mathrm{P}$ gene in HEX fluorescence channel as an internal control for parallel evaluation of sample quality/quantity and the test performance.

Both reverse transcription and LAMP reactions take place at $60^{\circ} \mathrm{C}$ using the M-MLV Reverse Transcriptase and Bst DNA polymerase. During the amplification, dual labeled PNA probes can be incorporated into the amplification products. Upon the incorporation, fluorescence is generated and can be monitored by the fluorescence reader on the real-time PCR detection platforms in a real time fashion.

The analytical specificity of the assay. The analytical specificity of the test was evaluated using 35 microorganisms shown in Table 2 which are frequently found in the human respiratory tract spiked in clinical negative nasopharyngeal (NP) swab specimen at concentrations of $10^{6} \mathrm{CFU} / \mathrm{mL}$ or higher for bacteria and $10^{5} \mathrm{pfu} / \mathrm{mL}$ or higher for viruses. In addition, RNA isolate from the SARS-CoV-2 negative human nasal wash was tested for specificity against the human normal nasal microflora. No detectable amplification curve was observed in FAM detection channel for SARS-CoV-2 ORF1ab and N genes, whereas the internal control RNase $\mathrm{P}$ in HEX detection channel did show $100 \%$ detection rate as expected in all three (3) test replicates for all organisms as well as for the nasal wash. Those results showed that the exclusivity of the assay against the microorganisms tested in this study is $100 \%$ ( $0 \%$ of false positivity) for both ORF1ab and N gene amplicon sets. Results are summarized in Table 2.

Clinical performance of the assay against commercial Real-time PCR test. A clinical evaluation of the PNA RT-LAMP assay was performed that evaluating a total of 270 blinded clinical NP swab specimens including 70 SARS-CoV-2 positive and 200 negative individual, leftover, de-identified specimens collected in the Chungnam National University Hospital which were previously tested using commercially available FDA EUA authorized real-time PCR test targeting SARS-CoV-2 specific RdRp and E genes (PowerCheck 2019-nCoV realtime PCR kit, Kogene Biotech). Positive samples were divided into 2 groups based on the Ct values exhibited on the real-time PCR: 1) High positives: a total of 44 samples which both targets showed $\mathrm{Ct} \leq 30$; 2) Low positives: a total of 26 samples which at least one of the targets showed $\mathrm{Ct}>30$. Both clinical sensitivity and specificity of the PNA RT-LAMP assay against the real-time PCR test result were confirmed to be $100 \%$ for both positive groups (Sensitivity 95\% CI: 94.80\%-100.00\%; Specificity 95\% CI: 98.10\%-100.00\%). Mean Ct values of the high and low positive groups on the real-time PCR were 22.95 and 31.53 for RdRp; 22.62 and 31.42 for N gene, while mean Tt (Threshold time) on the RT LAMP were 10.18 and 18.28 for ORF1ab; 9.39 and 15.56 for N gene, respectively. This result showed that the clinical performances of the 2 assays are identical, whereas the analysis time of RTLAMP is quite shorter than the real-time PCR. The results of the clinical evaluation are summarized in Table 3.

Comparative sensitivity of the PNA RT-LAMP assay and other EUA authorized SARS-CoV-2 molecular tests. The analytical sensitivity of the PNA RT-LAMP assay was evaluated using RNA extracts from heat-inactivated SARS-CoV-2 (USA-WA1/2020, ZeptoMetrix, USA) at tenfold dilution series spiked in clinical negative NP swabs comparing with commercially available FDA EUA authorized real-time RT-PCR test 


\begin{tabular}{|c|c|c|c|c|c|c|}
\hline & & \multicolumn{4}{|c|}{ Hit rate (\#detected/\#tested) } & \multirow{4}{*}{\begin{tabular}{|l} 
Result \\
Negative
\end{tabular}} \\
\hline & & \multicolumn{2}{|l|}{ A well } & \multicolumn{2}{|l|}{ B well } & \\
\hline \multicolumn{2}{|c|}{ Microorganisms } & ORF1ab & RNase P & $\mathbf{N}$ & RNase P & \\
\hline 1 & Human coronavirus 229E & $0 \%(0 / 3)$ & $100 \%(3 / 3)$ & $0 \%(0 / 3)$ & $100 \%(3 / 3)$ & \\
\hline 2 & Human coronavirus OC43 & $0 \%(0 / 3)$ & $100 \%(3 / 3)$ & $0 \%(0 / 3)$ & $100 \%(3 / 3)$ & Negative \\
\hline 3 & Human coronavirus HKU1 & $0 \%(0 / 3)$ & $100 \%(3 / 3)$ & $0 \%(0 / 3)$ & $100 \%(3 / 3)$ & Negative \\
\hline 4 & Human coronavirus NL63 & $0 \%(0 / 3)$ & $100 \%(3 / 3)$ & $0 \%(0 / 3)$ & $100 \%(3 / 3)$ & Negative \\
\hline 5 & SARS-coronavirus & $0 \%(0 / 3)$ & $100 \%(3 / 3)$ & $0 \%(0 / 3)$ & $100 \%(3 / 3)$ & Negative \\
\hline 6 & MERS-coronavirus & $0 \%(0 / 3)$ & $100 \%(3 / 3)$ & $0 \%(0 / 3)$ & $100 \%(3 / 3)$ & Negative \\
\hline 7 & Adenovirus type 1 & $0 \%(0 / 3)$ & $100 \%(3 / 3)$ & $0 \%(0 / 3)$ & $100 \%(3 / 3)$ & Negative \\
\hline 8 & Adenovirus type 2 & $0 \%(0 / 3)$ & $100 \%(3 / 3)$ & $0 \%(0 / 3)$ & $100 \%(3 / 3)$ & Negative \\
\hline 9 & Adenovirus type 3 & $0 \%(0 / 3)$ & $100 \%(3 / 3)$ & $0 \%(0 / 3)$ & $100 \%(3 / 3)$ & Negative \\
\hline 10 & Human Metapneumovirus & $0 \%(0 / 3)$ & $100 \%(3 / 3)$ & $0 \%(0 / 3)$ & $100 \%(3 / 3)$ & Negative \\
\hline 11 & Parainfluenza virus 1 & $0 \%(0 / 3)$ & $100 \%(3 / 3)$ & $0 \%(0 / 3)$ & $100 \%(3 / 3)$ & Negative \\
\hline 12 & Parainfluenza virus 2 & $0 \%(0 / 3)$ & $100 \%(3 / 3)$ & $0 \%(0 / 3)$ & $100 \%(3 / 3)$ & Negative \\
\hline 13 & Parainfluenza virus 3 & $0 \%(0 / 3)$ & $100 \%(3 / 3)$ & $0 \%(0 / 3)$ & $100 \%(3 / 3)$ & Negative \\
\hline 14 & Parainfluenza virus 4 & $0 \%(0 / 3)$ & $100 \%(3 / 3)$ & $0 \%(0 / 3)$ & $100 \%(3 / 3)$ & Negative \\
\hline 15 & Influenza A (H3N2) & $0 \%(0 / 3)$ & $100 \%(3 / 3)$ & $0 \%(0 / 3)$ & $100 \%(3 / 3)$ & Negative \\
\hline 16 & Influenza A (H1N1) & $0 \%(0 / 3)$ & $100 \%(3 / 3)$ & $0 \%(0 / 3)$ & $100 \%(3 / 3)$ & Negative \\
\hline 17 & Influenza B & $0 \%(0 / 3)$ & $100 \%(3 / 3)$ & $0 \%(0 / 3)$ & $100 \%(3 / 3)$ & Negative \\
\hline 18 & Enterovirus & $0 \%(0 / 3)$ & $100 \%(3 / 3)$ & $0 \%(0 / 3)$ & $100 \%(3 / 3)$ & Negative \\
\hline 19 & Respiratory syncytial virus & $0 \%(0 / 3)$ & $100 \%(3 / 3)$ & $0 \%(0 / 3)$ & $100 \%(3 / 3)$ & Negative \\
\hline 20 & Rhinovirus 1 & $0 \%(0 / 3)$ & $100 \%(3 / 3)$ & $0 \%(0 / 3)$ & $100 \%(3 / 3)$ & Negative \\
\hline 21 & Rhinovirus 14 & $0 \%(0 / 3)$ & $100 \%(3 / 3)$ & $0 \%(0 / 3)$ & $100 \%(3 / 3)$ & Negative \\
\hline 22 & Rhinovirus 7 & $0 \%(0 / 3)$ & $100 \%(3 / 3)$ & $0 \%(0 / 3)$ & $100 \%(3 / 3)$ & Negative \\
\hline 23 & Chlamydia pneumoniae & $0 \%(0 / 3)$ & $100 \%(3 / 3)$ & $0 \%(0 / 3)$ & $100 \%(3 / 3)$ & Negative \\
\hline 24 & Haemophilus influenzae & $0 \%(0 / 3)$ & $100 \%(3 / 3)$ & $0 \%(0 / 3)$ & $100 \%(3 / 3)$ & Negative \\
\hline 25 & Legionella pneumophila & $0 \%(0 / 3)$ & $100 \%(3 / 3)$ & $0 \%(0 / 3)$ & $100 \%(3 / 3)$ & Negative \\
\hline 26 & Mycobacterium tuberculosis & $0 \%(0 / 3)$ & $100 \%(3 / 3)$ & $0 \%(0 / 3)$ & $100 \%(3 / 3)$ & Negative \\
\hline 27 & Streptococcus pneumoniae & $0 \%(0 / 3)$ & $100 \%(3 / 3)$ & $0 \%(0 / 3)$ & $100 \%(3 / 3)$ & Negative \\
\hline 28 & Streptococcus pyogenes & $0 \%(0 / 3)$ & $100 \%(3 / 3)$ & $0 \%(0 / 3)$ & $100 \%(3 / 3)$ & Negative \\
\hline 29 & Bordetella pertussis & $0 \%(0 / 3)$ & $100 \%(3 / 3)$ & $0 \%(0 / 3)$ & $100 \%(3 / 3)$ & Negative \\
\hline 30 & Mycoplasma pneumoniae & $0 \%(0 / 3)$ & $100 \%(3 / 3)$ & $0 \%(0 / 3)$ & $100 \%(3 / 3)$ & Negative \\
\hline 31 & Candida albicans & $0 \%(0 / 3)$ & $100 \%(3 / 3)$ & $0 \%(0 / 3)$ & $100 \%(3 / 3)$ & Negative \\
\hline 32 & Pseudomonas aeruginosa & $0 \%(0 / 3)$ & $100 \%(3 / 3)$ & $0 \%(0 / 3)$ & $100 \%(3 / 3)$ & Negative \\
\hline 33 & Staphylococcus epidermis & $0 \%(0 / 3)$ & $100 \%(3 / 3)$ & $0 \%(0 / 3)$ & $100 \%(3 / 3)$ & Negative \\
\hline 34 & Streptococcus salivarius & $0 \%(0 / 3)$ & $100 \%(3 / 3)$ & $0 \%(0 / 3)$ & $100 \%(3 / 3)$ & Negative \\
\hline 35 & Staphylococcus aureus & $0 \%(0 / 3)$ & $100 \%(3 / 3)$ & $0 \%(0 / 3)$ & $100 \%(3 / 3)$ & Negative \\
\hline 36 & Human nasal wash & $0 \%(0 / 3)$ & $100 \%(3 / 3)$ & $0 \%(0 / 3)$ & $100 \%(3 / 3)$ & Negative \\
\hline
\end{tabular}

Table 2. Summary of cross reactivity study. RNA extracts from all tested microorganisms as well as direct human nasal wash were confirmed to not cross-react with PNA RT-LAMP assay.

(SS-9930, Seasun Biomaterials) and Colorimetric LAMP SARS-CoV-2 assay (E2019S, NEB). All three methods showed identical analytical sensitivities which exhibited the lowest detection limit of approximately 1 genomic copy of SARS-CoV-2 per microliter of RNA extract, indicating that the analytical sensitivity of the PNA RTLAMP assay was comparable to the real-time PCR and traditional colorimetric LAMP methods (Table 4, Supplementary Fig. S1).

For further evaluation, positive detection rates of PNA RT-LAMP and Colorimetric LAMP assays were evaluated using 15 clinical individual positive NP swabs including five (5) high positives which exhibited Ct values up to 30 cycles, five (5) moderate positives which exhibited Ct values between 31 to 34 cycles, and five (5) low positives which exhibited Ct values higher than 35 cycles for both SARS-CoV-2 ORF1ab and N genes that were previously identified using the Real-time RT PCR test (SS-9930, Seasun Biomaterials). The PNA RT-LAMP assay successfully detected all 15 samples from the three positive groups whereas the colorimetric LAMP test has missed 2 low positives which exhibited Ct values over 37 cycles for both ORF1ab and $\mathrm{N}$ genes on the real-time PCR assay (Table 5, Supplementary Fig. S2).

Those results show that the sensitivity of the PNA RT-LAMP assay is higher than the colorimetric LAMP assay and identical to the real-time PCR method even testing the low positive samples showed late amplification rates on the real-time PCR method. 


\begin{tabular}{|c|c|c|c|c|}
\hline \multicolumn{5}{|c|}{$\begin{array}{l}\text { (a) PNA RT-LAMP test AQ-TOP COVID-19 rapid detection kit } \\
\text { plus showed } 94.87-100.00 \%(95 \% \text { CI }) \text { of clinical sensitivity and } \\
98.17-100.00 \%(95 \% \mathrm{CI}) \text { of clinical sensitivity when testing against the } \\
\text { real-time PCR test }\end{array}$} \\
\hline & \multicolumn{4}{|c|}{ Comparator assay (Real-time PCR test) } \\
\hline & Positive & Negative & \multicolumn{2}{|c|}{ Total } \\
\hline \multicolumn{5}{|c|}{ AQ-TOP ${ }^{\text {mit }}$ COVID-19 Rapid Detection Kit Plus } \\
\hline Positive & 70 & 0 & \multicolumn{2}{|l|}{70} \\
\hline Negative & 0 & 200 & \multicolumn{2}{|l|}{200} \\
\hline Total & 70 & 200 & \multicolumn{2}{|l|}{270} \\
\hline Clinical sensitivity & \multicolumn{4}{|c|}{ 100\% (70/70); 95\% CI 94.87-100.00\% } \\
\hline Clinical specificity & \multicolumn{4}{|c|}{$100 \%$ (200/200); $95 \%$ CI 98.17-100.00\% } \\
\hline \multicolumn{5}{|c|}{ (b) $\mathrm{Ct}$ and $\mathrm{Tt}$ comparison of clinical positive samples } \\
\hline & \multicolumn{2}{|c|}{ Mean Ct (real-time PCR) } & \multicolumn{2}{|c|}{ Mean Tt (RT LAMP) } \\
\hline & ORFlab & N gene & RdRp & N gene \\
\hline High positives & 22.95 & 22.62 & 10.18 & 9.39 \\
\hline Low positives & 31.53 & 31.42 & 18.28 & 15.56 \\
\hline
\end{tabular}

Table 3. Summary of clinical evaluation results.

\begin{tabular}{|c|c|c|c|c|c|c|c|c|c|c|c|c|c|}
\hline \multirow[b]{3}{*}{ RNA concentration } & \multicolumn{5}{|c|}{ Real-time RT-PCR } & \multicolumn{5}{|c|}{ PNA RT-LAMP } & \multicolumn{3}{|c|}{ Colorimetric LAMP } \\
\hline & \multicolumn{2}{|c|}{ ORFlab } & \multicolumn{2}{|l|}{$\mathrm{N}$ gene } & \multirow[b]{2}{*}{ Result } & \multicolumn{2}{|c|}{ ORF1ab } & \multicolumn{2}{|l|}{$\mathrm{N}$ gene } & \multirow[b]{2}{*}{ Result } & \multicolumn{2}{|c|}{ N/E genes } & \multirow[b]{2}{*}{ Result } \\
\hline & Hit rate & Mean Ct & Hit rate & Mean Ct & & Hit rate & Mean Tt & Hit rate & Mean Tt & & Hit rate & Color & \\
\hline $10,000 \mathrm{cp} / \mu \mathrm{L}$ & $5 / 5$ & 21.14 & $5 / 5$ & 20.12 & Positive & $5 / 5$ & 7.10 & $5 / 5$ & 6.96 & Positive & $5 / 5$ & Yellow & Positive \\
\hline $1,000 \mathrm{cp} / \mu \mathrm{L}$ & $5 / 5$ & 24.68 & $5 / 5$ & 25.25 & Positive & $5 / 5$ & 8.42 & $5 / 5$ & 7.57 & Positive & $5 / 5$ & Yellow & Positive \\
\hline $100 \mathrm{cp} / \mu \mathrm{L}$ & $5 / 5$ & 28.99 & $5 / 5$ & 28.54 & Positive & $5 / 5$ & 10.66 & $5 / 5$ & 9.65 & Positive & $5 / 5$ & Yellow & Positive \\
\hline $10 \mathrm{cp} / \mu \mathrm{L}$ & $5 / 5$ & 32.30 & $5 / 5$ & 32.65 & Positive & $5 / 5$ & 11.39 & $5 / 5$ & 11.37 & Positive & $5 / 5$ & Yellow & Positive \\
\hline $1 \mathrm{cp} / \mu \mathrm{L}$ & $5 / 5$ & 36.65 & $5 / 5$ & 36.11 & Positive & $5 / 5$ & 12.19 & $5 / 5$ & 12.80 & Positive & $5 / 5$ & Yellow & Positive \\
\hline $0.1 \mathrm{cp} / \mu \mathrm{L}$ & $0 / 5$ & ND & $0 / 5$ & ND & Negative & $0 / 5$ & $\mathrm{ND}$ & $0 / 5$ & ND & Negative & $0 / 5$ & Red & Negative \\
\hline
\end{tabular}

Table 4. Comparative analysis of RT-LAMP, real-time PCR and colorimetric LAMP assays. All three methods exhibited the lowest detection limits capable of detecting SARS-CoV-2 genomic RNA of 1 genomic copy (cp) per $\mu \mathrm{L}$ of RNA extract. All three assays could not detect dilution series contain $0.1 \mathrm{cp} / \mu \mathrm{L}$ of SARS-CoV-2 RNA.

PNA RT-LAMP testing on a portable isothermal amplifier. Finally, we tested the 15 clinical high, moderate, low positive NP swabs on the PNA RT-LAMP test using a portable isothermal amplifier SMARTAMP (SS-7010, Seasun Biomaterials) which could collect fluorescence signals of FAM and HEX (excitation at 490-540 and emission at 515-555) reporter dyes in a real-time manner. The device is fully portable and compatible with tablet computers with an easy-to-use operating system that can be applicable at POC testing (Fig. 1). All 15 samples with various viral loads were detectable $100 \%$ within 15 min when testing with the same run condition as in the real-time PCR instrument (Table 5). This data shows that the PNA RT-LAMP assay can be applicable at POC testing even further evaluations with increasing clinical sample numbers are required.

\section{Discussion}

Here, we developed and evaluated analytical, clinical performances of PNA based RT-LAMP assay targeting ORF1 ab and N genes of SARS-CoV-2. However, the LAMP has been known as having high and rapid amplification efficiency which makes its analytical sensitivity comparable to real-time PCR, high rate of false positivity while testing field clinical samples has been reported on the strength of the result interpretation method based on $\mathrm{pH}$ dependent colorimetric visualization ${ }^{28,29}$. Since the colorimetric display of traditional LAMP is based on the principle of color change reaction of $\mathrm{pH}$ indicators such as phenol red $\mathrm{d}^{30}$, the result is significantly affected by remnants from nucleic acid extraction reagents contained in the clinical sample elutes as well as the LAMP reaction buffers and enzyme contents ${ }^{31}$. To overcome those issues, we applied dual-labeled PNA as a detection probe in LAMP reaction for fluorescence detection of the amplification product in a real-time manner. PNA has been reported that having superior specificity against its template nucleic acid with its neutrally charged peptide backbone nature which does not have a nonspecific binding affinity with minus charged natural phosphate backbone of the template nucleic acid $^{22,32}$. The un-cleavable peptide backbone also reduces the risk of non-specific signal production as a result of thermal degradation during long-term incubation at elevated temperatures.

We confirmed that the PNA RP-LAMP assay does not cross-react with non-target microorganisms at high concentrations. Also, both clinical specificity and sensitivity against real-time RT PCR assay showed $100 \%$ of 


\begin{tabular}{|c|c|c|c|c|c|c|c|c|c|c|c|c|c|c|c|c|c|c|c|}
\hline & & \multirow{2}{*}{\multicolumn{5}{|c|}{ Real-time RT-PCR }} & \multicolumn{10}{|c|}{ PNA RT-LAMP } & \multirow{3}{*}{\multicolumn{3}{|c|}{\begin{tabular}{|l|} 
Colorimetric LAMP \\
N/E genes
\end{tabular}}} \\
\hline & & & & & & & \multicolumn{5}{|c|}{ Testing on CFX-96 } & \multicolumn{5}{|c|}{ Testing on SMARTAMP } & & & \\
\hline & & ORF & & N gene & & & ORF1 & & N gen & & & ORF1 & & N ger & & & & & \\
\hline \multicolumn{2}{|l|}{ Sample } & $\mathrm{Ct}$ & Hit rate & $\mathrm{Ct}$ & Hit rate & Result & $\mathrm{Tt}$ & Hit rate & $\mathrm{Tt}$ & Hit rate & Result & $\mathrm{Tt}$ & Hit rate & $\mathrm{Tt}$ & Hit rate & Result & Color & Hit rate & Result \\
\hline \multirow{5}{*}{$\begin{array}{l}\text { High } \\
\text { posi- } \\
\text { tives }\end{array}$} & 1 & 23.5 & \multirow{5}{*}{$\begin{array}{l}5 / 5 \\
(100 \%)\end{array}$} & 22.7 & \multirow{5}{*}{$\begin{array}{l}5 / 5 \\
(100 \%)\end{array}$} & Positive & 9.0 & \multirow{5}{*}{$\begin{array}{l}5 / 5 \\
(100 \%)\end{array}$} & 7.5 & \multirow{5}{*}{$\begin{array}{l}5 / 5 \\
(100 \%)\end{array}$} & Positive & 8.9 & \multirow{5}{*}{$\begin{array}{l}5 / 5 \\
(100 \%)\end{array}$} & 8.1 & \multirow{5}{*}{$\begin{array}{l}5 / 5 \\
(100 \%)\end{array}$} & Positive & Yellow & \multirow{5}{*}{$\begin{array}{l}5 / 5 \\
(100 \%)\end{array}$} & Positive \\
\hline & 2 & 26.9 & & 25.7 & & Positive & 9.0 & & 7.5 & & Positive & 9.2 & & 8.0 & & Positive & Yellow & & Positive \\
\hline & 3 & 20.5 & & 19.2 & & Positive & 8.5 & & 6.8 & & Positive & 8.4 & & 7.2 & & Positive & Yellow & & Positive \\
\hline & 4 & 21.4 & & 20.3 & & Positive & 8.1 & & 6.6 & & Positive & 8.6 & & 6.5 & & Positive & Yellow & & Positive \\
\hline & 5 & 27.3 & & 26.3 & & Positive & 9.6 & & 8.1 & & Positive & 9.2 & & 7.5 & & Positive & Yellow & & Positive \\
\hline \multirow{5}{*}{$\begin{array}{l}\text { Moder- } \\
\text { ate posi- } \\
\text { tives }\end{array}$} & 1 & 33.6 & \multirow{5}{*}{$\begin{array}{l}5 / 5 \\
(100 \%)\end{array}$} & 31.71 & \multirow{5}{*}{$\begin{array}{l}5 / 5 \\
(100 \%)\end{array}$} & Positive & 14.2 & \multirow{5}{*}{$\begin{array}{l}5 / 5 \\
(100 \%)\end{array}$} & 11.1 & \multirow{5}{*}{$\begin{array}{l}5 / 5 \\
(100 \%)\end{array}$} & Positive & 13.8 & \multirow{5}{*}{$\begin{array}{l}5 / 5 \\
(100 \%)\end{array}$} & 10.5 & \multirow{5}{*}{$\begin{array}{l}5 / 5 \\
(100 \%)\end{array}$} & Positive & Yellow & \multirow{5}{*}{$\begin{array}{l}5 / 5 \\
(100 \%)\end{array}$} & Positive \\
\hline & 2 & 34.5 & & 33.3 & & Positive & 14.3 & & 11.0 & & Positive & 14.5 & & 12.3 & & Positive & Yellow & & Positive \\
\hline & 3 & 33.9 & & 32.0 & & Positive & 13.8 & & 10.9 & & Positive & 14.0 & & 11.6 & & Positive & Yellow & & Positive \\
\hline & 4 & 33.4 & & 31.5 & & Positive & 13.2 & & 9.9 & & Positive & 13.5 & & 10.4 & & \begin{tabular}{|l|} 
Positive \\
\end{tabular} & Yellow & & Positive \\
\hline & 5 & 33.5 & & 32.8 & & Positive & \begin{tabular}{|l|}
13.0 \\
\end{tabular} & & 11.1 & & Positive & \begin{tabular}{|l|}
13.8 \\
\end{tabular} & & 9.5 & & Positive & Yellow & & Positive \\
\hline \multirow{5}{*}{$\begin{array}{l}\text { Low } \\
\text { posi- } \\
\text { tives }\end{array}$} & 1 & 37.1 & \multirow{5}{*}{$\begin{array}{l}5 / 5 \\
(100 \%)\end{array}$} & 37.1 & \multirow{5}{*}{$\begin{array}{l}5 / 5 \\
(100 \%)\end{array}$} & Positive & 24.0 & \multirow{5}{*}{$\begin{array}{l}5 / 5 \\
(100 \%)\end{array}$} & 12.3 & \multirow{5}{*}{$\begin{array}{l}5 / 5 \\
(100 \%)\end{array}$} & Positive & 22.5 & \multirow{5}{*}{$\begin{array}{l}5 / 5 \\
(100 \%)\end{array}$} & 14.1 & \multirow{5}{*}{$\begin{array}{l}5 / 5 \\
(100 \%)\end{array}$} & Positive & Red & \multirow{5}{*}{$\begin{array}{l}3 / 5 \\
(60 \%)\end{array}$} & $\begin{array}{l}\text { Nega- } \\
\text { tive }\end{array}$ \\
\hline & 2 & 37.1 & & 35.7 & & Positive & 15.6 & & 12.9 & & Positive & 17.2 & & 13.6 & & Positive & Yellow & & Positive \\
\hline & 3 & 36.5 & & 35.8 & & Positive & 22.1 & & 11.7 & & Positive & 20.1 & & 14.3 & & Positive & Yellow & & Positive \\
\hline & 4 & 36.1 & & 35.5 & & Positive & 14.8 & & 15.0 & & Positive & 16.3 & & 11.5 & & Positive & Yellow & & Positive \\
\hline & 5 & 37.2 & & 36.6 & & Positive & 22.6 & & 13.7 & & Positive & 23.4 & & 12.5 & & Positive & Red & & $\begin{array}{l}\text { Nega- } \\
\text { tive }\end{array}$ \\
\hline
\end{tabular}

Table 5. Comparative table of clinical positive samples testing. 15 samples of high, moderate, and low positive groups exhibited positive amplification signals when tested using the PNA RT-LAMP assay on both CFX-96 real-time PCR detection system and a portable isothermal amplifier SMARTAMP. Colors of all 10 samples of high and moderate positive groups turned into yellow or orange indicating the amplification of SARS-CoV-2 targets, while two (2) low positive NP swabs (sample \#1, 5 bold in the table) out of the five (5) could not be detected as positive.

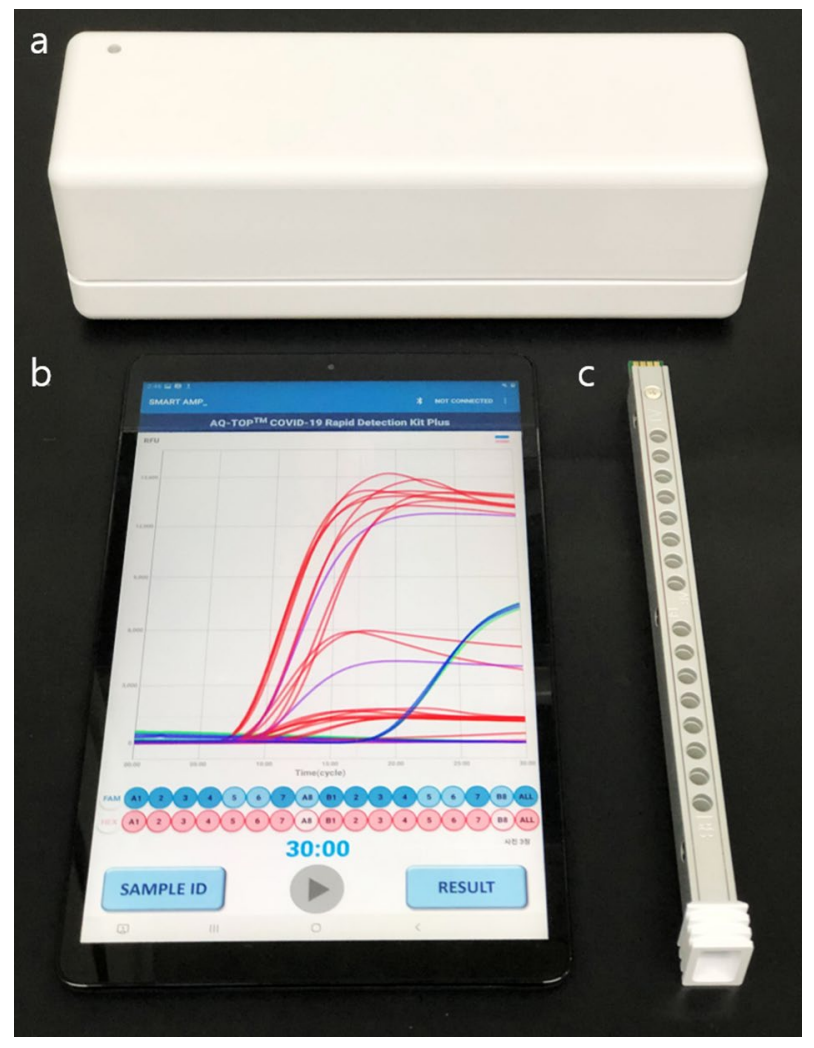

Figure 1. (a) A portable isothermal amplifier, (b) tablet computer with an operating system and c) 16 well heating plate applicable to the amplifier. 
accuracies. The above results showed that PNA RT-LAMP assay employs high enough analytical specificity as well as the clinical performances are comparable to the gold standard real-time PCR method.

Results of the sensitivity testing using tenfold dilution series of SARS-CoV-2 inactivated isolate demonstrated the potential of the PNA RT-LAMP test could detect $\sim 1$ copy of template RNA per microliter of the sample within approximately $12 \mathrm{~min}$, however, the next tenfold dilution series contain approximately 0.1 copy of the SARS$\mathrm{CoV}-2$ genomic material could not be detected even in reaction up to $30 \mathrm{~min}$. Practically, every tenfold dilution series in real-time PCR exhibit the Ct values of 3.3 apart while PNA RT-LAMP (in this study) exhibited one (1) minute lateness for every tenfold dilution series. According to these practices, the dilution series contain $\sim 0.1$ genomic copies could be detected on PNA RT-LAMP assay although it couldn't. Our assumption on this was the most amount of Bst polymerase has already been consumed during amplification of an internal control RNase $\mathrm{P}$, and not enough concentration of active enzymes remained for amplification of the SARS-CoV-2 in extremely low concentration. Because RNase P showed amplification signals at around 15-20 Tt on the RT LAMP while the SARS-CoV-2 targets near to the test LOD showed Tt around 12-13 (Supplementary Fig. S1). We observed that once RNase P amplification has started SARS-CoV-2 targets were not amplified. To test this assumption, we did further tests by increasing the Bst concentration up to tenfold, reducing the primer/probe concentrations of the internal control, however the expected result has not been obtained.

The final goal of this study was the development of a rapid molecular detection method that can be applicable at POC testing while having a comparable clinical performance with the gold standard real-time PCR method. We have confirmed that the PNA RT-LAMP assay can detect low positive samples contain a few copies of target RNA that exhibiting Ct values $>35$ on the real-time PCR although traditional LAMP has missed those samples. This result was reproducible on a both benchtop real-time PCR detection system and a portable isothermal amplification device.

However, the PNA RT-LAMP assay developed in this study is currently applicable with RNA extracts from clinical NP swabs, we have been working on optimization of the assay on use of saliva and direct NP swabs without an additional sample preparation step which is more suitable at POC testing and in low-resource settings.

\section{Methods}

Materials used in this study. 2×LAMP Master Mix contains M-MLV Reverse Transcriptase and Bst DNA polymerase were purchased from Elpis-Biotech, Korea. Oligonucleotides and dual labeled PNA probes were synthesized in Cosmo Genetech, Korea, and Panagene, Korea respectively. Heat inactivated SARS-CoV-2 isolate USA-WA1/2020 was purchased from ZeptoMetrix, USA. Strains of the microorganisms used in the specificity testing were purchased from Korean Bank of Pathogenic Viruses and the National Culture Collection for Pathogens, Korea.

Sequence analysis. A total of 12 primer and 2 PNA probe sequences were analyzed against 56,303 SARSCoV-2 genomic sequences contain whole genome information downloaded from GenBank (https://www.ncbi. nlm.nih.gov/) on CLC Main Workbench 9.5.2 with molecular biology tool "Find binding sites and create fragment".

Sample preparation. For analytical sensitivity study, RNAs were purified from $300 \mu \mathrm{L}$ of NP swabs spiked in tenfold dilutions series of SARS-CoV-2 inactivated isolate (ZeptoMetrix, USA-WA1/2020) using TOP Viral DNA/RNA extraction kit (Seasun Biomaterials, SS-1300) according to the manufacturer's instructions and eluted in $30 \mu \mathrm{L}$ of elution buffer included in the kit. Genomic copies per $\mu \mathrm{L}$ were previously quantified using NanoDrop values of the nucleic acid extract of undiluted SARS-CoV-2 isolate as a formula in below:

$$
\text { Genomic copies }=\frac{n g \text { of single stranded RNA } \times \text { Avagadro' } s \text { constant }\left(6.02 \times 10^{23}\right)}{\text { Length of nucleotide } \times 10^{9} \times 325 \text { Daltons }}
$$

The size of the SARS-CoV-2 reference genome (NCBI Reference Sequence: NC_045512.2) is assumed to be 29,903 bp ss-RNA was used in the calculations. For comparative clinical sensitivity and clinical evaluation, $300 \mu \mathrm{L}$ clinical positive NP swab specimens were processed using PANAMAX48 viral DNA/RNA extraction kit (Panagene, PNAK 1001) on PANAMAX nucleic acid automated extractor following the manufacturer's instructions. Each RNA isolate was used immediately after the extraction.

PNA RT-LAMP amplification and detection. PNA RT-LAMP test was performed with a total of $30 \mu \mathrm{L}$ of total reaction volume using $15 \mu \mathrm{L}$ of reaction buffer, $1 \mu \mathrm{L}$ of enzyme mix, $4 \mu \mathrm{L}$ of the reaction mix, and $10 \mu \mathrm{L}$ of template RNA on CFX-96 real-time PCR detection system or SMARTAMP portable isothermal amplifier with the run condition of 60 degrees Celsius for $30 \mathrm{~min}$ with fluorescence signal collection at every $1 \mathrm{~min}$. Samples that exhibited positive amplification signals within $30 \mathrm{~min}$ on FAM fluorescence channel in at least one reaction well was defined as positives. Samples that did not produce positive FAM signal while HEX detection channel regarding endogenous quality control RNase P produced amplification curve were defined as negatives.

Clinical evaluation. RNAs were extracted from $200 \mu \mathrm{L}$ of individual, leftover, de-identified nasopharyngeal swab specimens collected in the Chungnam National University Hospital previously tested with FDA authorized under EUA SARS-CoV-2 real-time PCR assay PowerCheck 2019-nCoV real-time PCR kit (Kogene Biotech, Korea) targeting SARS-CoV-2 specific RdRp and E genes. $10 \mu \mathrm{L}$ of RNA extracts were tested for each reaction mixtures of the PNA RT-LAMP assay in a blinded manner on the CFX-96 real-time PCR detection system. Clinical accuracies were calculated by using the standard method in the base of CI $95 \%{ }^{33}$. 
Sensitivity testing. RNA extracts were tested with five (5) individual extraction replicates on the PNA RT-LAMP assay, SARS-CoV-2 real-time RT-PCR assay U-TOP COVID-19 Detection Kit (SS-9930, Seasun Biomaterials) targets ORF1an/RdRp and N gene of SARS-CoV-2 with human RNase P in a single tube; and SARSCoV-2 Rapid Colorimetric LAMP Assay Kit (E2019S, NEB) targets SARS-CoV-2 E/N genes in one tube, and rActin in a separate tube for the quality control according to the instructions provided from the manufacturers. Results were interpreted according to the instructions supplied from the manufacturers as briefly the samples which exhibited Ct values less than 38 for at least one SARS-CoV-2 target gene were defined as positives for the real-time PCR assay, and samples which color of the reaction mixture turned into yellow or light orange from red after completion of the LAMP reaction were defined as positives for the colorimetric LAMP assay. Real-time PCR was performed on the CFX-96 real-time PCR detection system, SARS-CoV-2 Rapid Colorimetric LAMP Assay was performed using ABI2720 thermal cycler (Applied Biosystems, USA). PNA RT-LAMP test was performed on both CFX-96 real-time PCR detection system and SMARTAMP isothermal amplifier with the same experimental condition.

Source of clinical specimens and ethnics statement. Clinical samples collected in Chungnam National University Hospital at the first wave of COVID-19 (February to April 2020) in the Republic of Korea were used in this study. Handling and analysis of the clinical samples were approved by the Institutional Review Board (IRB. CNUH2020-06-123) of Chungnam National University Hospital. Informed consent was obtained from all participants and the research was performed on anonymized, de-identified RNA samples following the Declaration of Helsinki.

Received: 25 May 2021; Accepted: 5 October 2021

Published online: 14 October 2021

\section{References}

1. Hu, T. et al. A comparison of COVID-19, SARS and MERS. PeerJ 8, e9725. https://doi.org/10.7717/peerj.9725 (2020).

2. Liu, J. et al. A comparative overview of COVID-19, MERS and SARS: Review article. Int. J. Surg. 81, 1-8. https://doi.org/10.1016/j. ijsu.2020.07.032 (2020).

3. Huang, W. E. et al. RT-LAMP for rapid diagnosis of coronavirus SARS-CoV-2. Microb. Biotechnol. 13, 950-961. https://doi.org/ 10.1111/1751-7915.13586(2020)

4. Xiong, D. et al. Rapid detection of SARS-CoV-2 with CRISPR-Cas12a. PLoS Biol. 18, e3000978. https://doi.org/10.1371/journal. pbio.3000978 (2020).

5. Chou, P. H., Lin, Y. C., Teng, P. H., Chen, C. L. \& Lee, P. Y. Real-time target-specific detection of loop-mediated isothermal amplification for white spot syndrome virus using fluorescence energy transfer-based probes. J. Virol. Methods 173, 67-74. https://doi. org/10.1016/j.jviromet.2011.01.009 (2011).

6. Fowler, V. L. et al. A highly effective reverse-transcription loop-mediated isothermal amplification (RT-LAMP) assay for the rapid detection of SARS-CoV-2 infection. J. Infect. 82, 117-125. https://doi.org/10.1016/j.jinf.2020.10.039 (2021).

7. Haq, F. et al. Reverse transcriptase loop-mediated isothermal amplification (RT-LAMP)-based diagnosis: A potential alternative to quantitative real-time PCR based detection of the novel SARS-COV-2 virus. Saudi J. Biol. Sci. 28, 942-947. https://doi.org/10. 1016/j.sjbs.2020.10.064 (2021).

8. Zhang, Y. \& Tanner, N. A. Development of multiplexed reverse-transcription loop-mediated isothermal amplification for detection of SARS-CoV-2 and influenza viral RNA. Biotechniques 70, 167-174. https://doi.org/10.2144/btn-2020-0157 (2021).

9. Hardinge, P. \& Murray, J. A. H. Reduced false positives and improved reporting of loop-mediated isothermal amplification using quenched fluorescent primers. Sci. Rep. 9, 7400. https://doi.org/10.1038/s41598-019-43817-z (2019).

10. Kollenda, H. et al. Poor diagnostic performance of a species-specific loop-mediated isothermal amplification (LAMP) platform for malaria. Eur. J. Microbiol. Immunol. 8, 112-118. https://doi.org/10.1556/1886.2018.00020 (2018).

11. Moonga, L. C. et al. Development of a multiplex loop-mediated isothermal amplification (LAMP) method for simultaneous detection of spotted fever group rickettsiae and malaria parasites by dipstick DNA chromatography. Diagnostics https://doi.org/ $10.3390 /$ diagnostics10110897 (2020).

12. Liang, C. et al. Multiplex loop-mediated isothermal amplification detection by sequence-based barcodes coupled with nicking endonuclease-mediated pyrosequencing. Anal. Chem. 84, 3758-3763. https://doi.org/10.1021/ac3003825 (2012).

13. Rodriguez-Manzano, J. et al. Reading out single-molecule digital RNA and DNA isothermal amplification in nanoliter volumes with unmodified camera phones. ACS Nano 10, 3102-3113. https://doi.org/10.1021/acsnano.5b07338 (2016).

14. Aoki, M. N. et al. Colorimetric RT-LAMP SARS-CoV-2 diagnostic sensitivity relies on color interpretation and viral load. Sci. Rep. 11, 9026. https://doi.org/10.1038/s41598-021-88506-y (2021).

15. Kitajima, H. et al. Clinical COVID-19 diagnostic methods: Comparison of reverse transcription loop-mediated isothermal amplification (RT-LAMP) and quantitative RT-PCR (qRT-PCR). J. Clin. Virol. 139, 104813. https://doi.org/10.1016/j.jcv.2021.104813 (2021).

16. Freire-Paspuel, B. \& Garcia-Bereguiain, M. A. Low clinical performance of the Isopollo COVID-19 detection kit (M Monitor, South Korea) for RT-LAMP SARS-CoV-2 diagnosis: A call for action against low quality products for developing countries. Int. J. Infect. Dis. 104, 303-305. https://doi.org/10.1016/j.ijid.2020.12.088 (2021).

17. Khan, M. et al. Comparative evaluation of the LAMP assay and PCR-based assays for the rapid detection of Alternaria solani. Front. Microbiol. 9, 2089. https://doi.org/10.3389/fmicb.2018.02089 (2018).

18. Inaba, M. et al. Diagnostic accuracy of LAMP versus PCR over the course of SARS-CoV-2 infection. Int. J. Infect. Dis. 107, 195-200. https://doi.org/10.1016/j.ijid.2021.04.018 (2021).

19. Hur, D., Kim, M. S., Song, M., Jung, J. \& Park, H. Detection of genetic variation using dual-labeled peptide nucleic acid (PNA) probe-based melting point analysis. Biological procedures online 17, 14. https://doi.org/10.1186/s12575-015-0027-5 (2015).

20. Choi, J. J., Jang, M., Kim, J. \& Park, H. Highly sensitive PNA array platform technology for single nucleotide mismatch discrimination. J. Microbiol. Biotechnol. 20, 287-293 (2010).

21. Porcheddu, A. \& Giacomelli, G. Peptide nucleic acids (PNAs), a chemical overview. Curr. Med. Chem. 12, 2561-2599. https://doi. org/10.2174/092986705774370664(2005). 
22. Choi, W. S. et al. Peptide nucleic acid (PNA)-enhanced specificity of a dual-target real-time quantitative polymerase chain reaction (RT-qPCR) assay for the detection and differentiation of SARS-CoV-2 from related viruses. Diagnostics https://doi.org/10.3390/ diagnostics10100775 (2020).

23. Das, A. \& Pradhan, B. Evolution of peptide nucleic acid with modifications of its backbone and application in biotechnology. Chem. Biol. Drug Des. 97, 865-892. https://doi.org/10.1111/cbdd.13815 (2021).

24. Tarumoto, N. et al. A novel peptide nucleic acid- and loop-mediated isothermal amplification assay for the detection of mutations in the 23S rRNA gene of Treponema pallidum. J. Med. Microbiol. 69, 1339-1345. https://doi.org/10.1099/jmm.0.001275 (2020).

25. Moccia, M. et al. Paper-based electrochemical peptide nucleic acid (PNA) biosensor for detection of miRNA-492: A pancreatic ductal adenocarcinoma biomarker. Biosens. Bioelectron. 165, 112371. https://doi.org/10.1016/j.bios.2020.112371 (2020).

26. Saarbach, J., Sabale, P. M. \& Winssinger, N. Peptide nucleic acid (PNA) and its applications in chemical biology, diagnostics, and therapeutics. Curr. Opin. Chem. Biol. 52, 112-124. https://doi.org/10.1016/j.cbpa.2019.06.006 (2019).

27. Oliveira, R., Almeida, C. \& Azevedo, N. F. Detection of microorganisms by fluorescence in situ hybridization using peptide nucleic acid. Methods Mol. Biol. 2105, 217-230. https://doi.org/10.1007/978-1-0716-0243-0_13 (2020).

28. Hsieh, K., Mage, P. L., Csordas, A. T., Eisenstein, M. \& Soh, H. T. Simultaneous elimination of carryover contamination and detection of DNA with uracil-DNA-glycosylase-supplemented loop-mediated isothermal amplification (UDG-LAMP). Chem. Commun. 50, 3747-3749. https://doi.org/10.1039/c4cc00540f (2014).

29. Suleman, E., Mtshali, M. S. \& Lane, E. Investigation of false positives associated with loop-mediated isothermal amplification assays for detection of Toxoplasma gondii in archived tissue samples of captive felids. J. Vet. Diagn. Investig. 28, 536-542. https:// doi.org/10.1177/1040638716659864 (2016).

30. Tanner, N. A., Zhang, Y. \& Evans, T. C. Jr. Visual detection of isothermal nucleic acid amplification using pH-sensitive dyes. Biotechniques 58, 59-68. https://doi.org/10.2144/000114253 (2015).

31. Poole, C. B. et al. Colorimetric tests for diagnosis of filarial infection and vector surveillance using non-instrumented nucleic acid loop-mediated isothermal amplification (NINA-LAMP). PLoS ONE 12, e0169011. https://doi.org/10.1371/journal.pone.0169011 (2017).

32. De Costa, N. T. \& Heemstra, J. M. Evaluating the effect of ionic strength on duplex stability for PNA having negatively or positively charged side chains. PLoS ONE 8, e58670. https://doi.org/10.1371/journal.pone.0058670 (2013).

33. Baratloo, A., Hosseini, M., Negida, A. \& El Ashal, G. Part 1: Simple definition and calculation of accuracy, sensitivity and specificity. Emergency 3, 48-49 (2015).

\section{Author contributions}

H.G.K. and S.S.L. designed and performed all experiments and evaluation tests. Y.S.K. performed the clinical evaluation. C.B. wrote the manuscript. H.G.K., S.S.L., Y.S.K., H.W.L., H.K.P. revised the manuscript. All authors approved the final version of the article and agree to be accountable for all aspects of the work.

\section{Competing interests}

$\mathrm{CB}, \mathrm{HGK}, \mathrm{SSL}, \mathrm{HWL}$ and HKP are employees of manufacturer of the RT LAMP assay described in this paper. The other authors declare no competing interests.

\section{Additional information}

Supplementary Information The online version contains supplementary material available at https://doi.org/ 10.1038/s41598-021-00041-y.

Correspondence and requests for materials should be addressed to C.B.-O.

Reprints and permissions information is available at www.nature.com/reprints.

Publisher's note Springer Nature remains neutral with regard to jurisdictional claims in published maps and institutional affiliations.

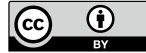

Open Access This article is licensed under a Creative Commons Attribution 4.0 International License, which permits use, sharing, adaptation, distribution and reproduction in any medium or format, as long as you give appropriate credit to the original author(s) and the source, provide a link to the Creative Commons licence, and indicate if changes were made. The images or other third party material in this article are included in the article's Creative Commons licence, unless indicated otherwise in a credit line to the material. If material is not included in the article's Creative Commons licence and your intended use is not permitted by statutory regulation or exceeds the permitted use, you will need to obtain permission directly from the copyright holder. To view a copy of this licence, visit http://creativecommons.org/licenses/by/4.0/.

(C) The Author(s) 2021, corrected publication 2021 